\title{
Some insights on the negative effect played by silylation of functionalized commercial silica in the direct synthesis of hydrogen peroxide
}

\author{
G. Blanco Brieva ${ }^{a^{*}}$, J. M. Campos-Martin ${ }^{a}$, M. P. de Frutos ${ }^{b}$ and J. L. G. Fierro ${ }^{a}$ \\ ${ }^{a}$ Sustainable Energy and Chemistry Group. Instituto de Catálisis y Petroleoquímica, CSIC, Marie Curie, 2 , \\ Cantoblanco, 28049 Madrid, Spain. \\ http://www.icp.csic.es/eqs \\ ${ }^{\mathrm{b}}$ Centro de Tecnología Repsol YPF, A-5, Km. 18, 28931 Móstoles, Spain.
}

\author{
*CORRESPONDING AUTHOR FOOTNOTE \\ Dr. Gema Blanco-Brieva, e-mail: gblanco@icp.csic.es \\ Fax: +34 915854760 \\ http://www.icp.csic.es/eqs
}

\begin{abstract}
The surface of sulfonic acid functionalized silica has been modified by silylation, using two silylating agents: $\left(\mathrm{CH}_{3}\right)_{3} \mathrm{SiCl}$ and $\mathrm{CF}_{3}\left(\mathrm{CF}_{2}\right)_{5}\left(\mathrm{CH}_{2}\right)_{2} \mathrm{Si}\left(\mathrm{CH}_{3}\right)_{2} \mathrm{Cl}$. The modified supports were characterized by different techniques. For these systems, BET specific area was found to decrease upon silylation, but the effect on other textural properties depends on the silylating agent used. Once modified the supports were taken to prepare catalysts by ionic exchange with a palladium salt. The palladium species ratio depends on the used support. Catalyst system of supported palladium nanoparticles deposited on $\mathrm{HSO}_{3}$-functionalized commercial silica produces hydrogen peroxide with a high yield by feeding a non-flammable $\mathrm{H}_{2} / \mathrm{O}_{2}$ mixture into a non-acidic catalyst-methanol suspension at $313 \mathrm{~K}$. However, catalysts prepared with silylated supports show a low yield to hydrogen peroxide, due to low $\mathrm{H}_{2} \mathrm{O}_{2}$ selectivity. The drop in the selectivity to hydrogen peroxide is related with the different proportion of palladium species present in silylated catalysts as compared to non silyled sample.
\end{abstract}

Keywords: $\mathrm{H}_{2} \mathrm{O}_{2}$, catalyst, direct synthesis, palladium, hydrophobic surface. 


\section{Introduction}

Hydrogen peroxide is a clean oxidizing and bleaching agent that is often used in many environmental applications for water purification/waste water treatments, as whitening agent, disinfectant and also as basic product of the chemical industry [1]. Currently, hydrogen peroxide is produced by standard anthraquinone process [2] however its cost is rather high as to be used for the production of bulk organic chemicals. Synthesis of hydrogen peroxide by direct reaction between $\mathrm{H}_{2}$ and $\mathrm{O}_{2}\left(\mathrm{H}_{2}+\mathrm{O}_{2} \rightarrow \mathrm{H}_{2} \mathrm{O}_{2}\right)$ can be a very attractive alternative to this process. Direct synthesis over Pd-based catalyst was first reported by Henkel and Webel in 1914 [3] and several studies have been issued since then [1 and references therein]. However this process could not be put into practice. There are two mayor drawbacks in this process: (i), the explosive limits of hydrogen/oxygen gas mixtures are very wide [4] and are further widened with increasing pressure; and (ii), other important problem is the limited selectivity to $\mathrm{H}_{2} \mathrm{O}_{2}$ yield reached in the direct synthesis reaction. In order to be out of the explosive region, mixtures of $\mathrm{H}_{2}$ and $\mathrm{O}_{2}$ should contain less than about $4 \%$ hydrogen.

Once formed, hydrogen peroxide decomposes slowly into water and oxygen. To delay or prevent the decomposition of $\mathrm{H}_{2} \mathrm{O}_{2}$, acids are often incorporated into the reaction medium which indeed takes places in the presence of bases [5]. Along the synthesis step, some catalyst poisons, i.e. halides, also delay the water formation reaction and accordingly increase the hydrogen peroxide selectivity [5-8]. Although halides show good selectivity for $\mathrm{H}_{2} \mathrm{O}_{2}$ formation, they also delay the hydrogen conversion [5,7]. This suggests that the halide ions are adsorbed on the metal centers that participate in the surface reaction between hydrogen and oxygen [6]. The presence of acid solutions and halide ions also favors solubilization of the active metal catalyst $[9,10]$, which results in deactivation of the catalyst. These problems can be avoided with the use of neutral solutions. Excellent yields have been reported using neutral solutions and heterogeneous catalysts consisting of sulfonic acid functionalized polystyrene resins $\left(\mathrm{PS}-\mathrm{SO}_{3} \mathrm{H}\right)$ [11-13]. When polyvinylbenzene-based catalysts are used, the process typically becomes diffusion-limited, and the polymer is swollen and deactivated in aggressive reaction media. Additionally, resin catalysts suffer from a lack of mechanical strength, thermal stability, and low specific areas. These drawbacks can be corrected by the use of silica functionalized with sulfonic groups $[15,16]$. These supports can be used in the 
direct synthesis of hydrogen peroxide [17]. A recent report indicates the strong effect of the surface modification on the selectivity to hydrogen peroxide [18].

One of the simplest approaches to modify the surface properties of silica substrates consists in the covalent anchorage of some organosilica chemical compounds. The silylation of porous and non-porous silica has been widely used in different fields [19]. For these two kinds of silica substrates the density of the active surface groups instead porosity determines the type and extent of functionalization. When the silica is silylated with a pure (alkyl)- or (alkoxy)chlorosilane, high reaction temperatures are required to complete the reaction. However, when a nucleophile is added in the vapour or liquid phase, the reaction temperature can be decreased towards room temperature [19]. The only option to silylate silica functionalized with sulfonic groups is the use of (methyl) chlorosilanes, because the presence of the nucleophile can interact with sulfonic groups. The reactions between (methyl) chlorosilanes and the surface of silica have been investigated in some detail [19,20]. Although the use of (methyl)chlorosilanes results in the formation of a hydrophobic surface, more hydrophobic surface can be obtained by modification with perfluorosilane; the surface becomes superrepellent towards a broad range of liquids, which include (salted) water, water emulsion, common organic liquids like glycerol and alkanes, and a variety of lubrication oils including ionic liquids, poly( $\alpha$-olefin), polydimethylsiloxane oils, etc. [21].

Based on these precedents, this work was undertake with the aim to study the effect of the surface modification of sulphonic acid functionalized silica by silylation and using these modified substrates to prepare Pd-based catalysts for the direct synthesis of $\mathrm{H}_{2} \mathrm{O}_{2}$ in nonacidic solutions. This surface modification results also in a more hydrophobic surface which is expected to be beneficial to minimize the extent of the secondary reactions accompanying the principal, wanted synthesis reaction.

\section{Experimental}

\section{Support Modification}

In a first step, to a suspension of functionalized commercial silica (Silycicle Tosic Acid) (10 $\mathrm{g})$ in toluene $(100 \mathrm{ml}),\left(\mathrm{CH}_{3}\right)_{3} \mathrm{SiCl}$ or $\mathrm{CF}_{3}\left(\mathrm{CF}_{2}\right)_{5}\left(\mathrm{CH}_{2}\right)_{2} \mathrm{Si}\left(\mathrm{CH}_{3}\right)_{2} \mathrm{Cl}$ was added dropwise. The suspension was stirred for $6 \mathrm{~h}$ under refluxing. The remaining solution was filtered off and the 
solid obtained was washed with toluene $(50 \mathrm{ml})$ and air-dried at $373 \mathrm{~K}$ for $12 \mathrm{~h}$. A scheme of the silylation procedure and samples labeling is shown in Scheme 1.

\section{Catalysis Preparation}

Modified support (10 g) was stirred with $125 \mathrm{~mL}$ of acetone. To this suspension, a palladium (II) acetate (Johnson Matthey) solution in acetone $(50 \mathrm{ml})$ was dropwise added. The suspension was stirred for $1 \mathrm{~h}$. The remaining solution was filtered off and the solid obtained was washed and air-dried at $333 \mathrm{~K}$ for $2 \mathrm{~h}$.

\section{Catalysts Characterization}

Thermogravimetric analyses of the silica supports were performed with a Perkin-Elmer TGS2 instrument, working at a heating rate of $10 \mathrm{~K} \mathrm{~min}^{-1}$ under nitrogen flow $\left(60 \mathrm{~mL} \mathrm{~min}^{-1}\right)$.

The textural properties of the supports (Table 1) were determined from the nitrogen adsorption-desorption isotherms recorded at $77 \mathrm{~K}$ with a Micromeritics TriStar 3000 apparatus. The samples were previously degassed at $423 \mathrm{~K}$ for $24 \mathrm{~h}$ under a vacuum $\left(10^{-4}\right.$ mbar) to ensure a clean dry surface, free of any loosely bound adsorbed species. The specific area of the samples were calculated by the BET method using the nitrogen adsorption data taken in the relative equilibrium pressure interval of $0.03<\mathrm{P} / \mathrm{P}^{0}<0.3$ and with a value of $0.162 \mathrm{~nm}^{2}$ for the cross-section of adsorbed nitrogen molecule. Pore distributions were calculated using the $\mathrm{BJH}$ model and taking data from the desorption branch of the isotherms.

X-ray photoelectron spectra (XPS) were acquired with a VG Escalab 200R spectrometer equipped with a hemispherical electron analyzer and an $\mathrm{Mg} \mathrm{K \alpha}(\mathrm{h} v=1253.6 \mathrm{eV})$ nonmonochromatic X-ray source. The samples were degassed in the pretreatment chamber at room temperature for $1 \mathrm{~h}$ prior to being transferred into the instrument's ultra-high vacuum analysis chamber. The $\mathrm{Pd} 3 \mathrm{~d}, \mathrm{O} 1 \mathrm{~s}, \mathrm{~S} 2 \mathrm{p}$ and $\mathrm{C} 1 \mathrm{~s}$ signals were scanned several times at pass energy of $50 \mathrm{eV}$ in order to obtain good signal-to-noise ratios. The binding energies (BE) were referenced to the $\mathrm{BE}$ of $\mathrm{C} 1 \mathrm{~s}$ core-level peak at $284.9 \mathrm{eV}$. The invariance of the peak shapes and widths at the beginning and end of the analyses indicated constant charge along measurements. The peaks were fitted by a non-linear least square fitting routine using a properly weighted sum of Lorentzian and Gaussian component curves after background subtraction. Surface atomic ratios were estimated from the areas of the peaks, normalized to silicon, and corrected using the corresponding sensitivity factors [22]. 


\section{Direct Hydrogen Peroxide Synthesis}

Catalysts were tested in the direct synthesis of $\mathrm{H}_{2} \mathrm{O}_{2}$. In a typical run, $1.6 \mathrm{~g}$ of the catalyst was placed inside an autoclave with $150 \mathrm{~g}$ of methanol and $\mathrm{HBr}(24 \mathrm{ppm})$ as promoter. The reactor was pressured with $\mathrm{N}_{2}$ to $9.5 \mathrm{MPa}$. The mixture was heated to $313 \mathrm{~K}$. Then, the reaction gas mixture was feed $\left(\mathrm{H}_{2}: \mathrm{O}_{2}: \mathrm{N}_{2}(3.6: 46.4: 50)\right)$ with a total flow of $2500 \mathrm{~mL}(\mathrm{~N}) \mathrm{min}^{-1}$ without stirring, and then stirring was started up (1500 rpm) to initiate the reaction. Hydrogen consumption was determined by GC-TCD using a Varian CP-4900 microGC device. Hydrogen peroxide and water concentrations were measured by iodometric and Karl-Fischer standard titrations, respectively.

\section{Results and discussion}

Thermogravimetric profiles showed that all samples lose a small amount of weight at low temperature $(<383 \mathrm{~K}$ ) (Figure 1), which is associated to desorption of adsorbed molecular water. Silylated samples showed a lower weight loss than the starting silica (SiCo), but no significant differences have been observed between silylated samples (Figure 1). Although this observation is indirect evidence, it could be argued that the silylated samples exhibit greater hydrophobicity than the original silica. Silylated supports showed a small weight loss around $500 \mathrm{~K}$, that can be attributed to desorption of residual solvent (toluene) employed in the silylation step. All TG profiles showed weight loss starting at around $573 \mathrm{~K}$, which is attributed to the decomposition of organic groups bonded to the silica surface (sulfonic and silylating groups) with an inflection point at $831 \mathrm{~K}$.

All the nitrogen adsorption-desorption isotherms belong to type IV of the IUPAC classification, which is characteristic of mesoporous materials (Figure 2). These isotherms also show a type $\mathrm{H} 1$ hysteresis loop located at relatively high pressures of the adsorbate, which is usually displayed by porous materials consisting of agglomerates or compact packing of nearly uniform, regularly ordered microspheres [23]. The silylation process brought about a large decrease in the nitrogen adsorption capacity, reflected in a decrease in the specific areas of all the samples (Table 1). Thus, the BET area of the original, nonfunctionalized (SiCo) sample $\left(251 \mathrm{~m}^{2} \mathrm{~g}^{-1}\right)$ decreases to approximately $201 \mathrm{~m}^{2} \mathrm{~g}^{-1}$ in silylated samples. Pore size distribution, as determined by applying the BJH model to the desorption branch of the nitrogen adsorption-desorption isotherms, also underwent some changes. The 
pore sizes of silylated samples increase with respect to the starting silica (SiCo). This effect can be attributed to changes in the surface of silica nanospheres as a consequence of the reaction between the silylating agent and surface hydroxyl groups, which makes the population of hydroxyl groups to decrease. In this partially grafted surface the attraction forces between particles becomes weakened and hence the average distance or pore volume between closer nanospheres slightly increases. The larger increase of pore volume for $\mathrm{SiCoCl}$ indicates a homogenous silylation of the surface of this sample (Table 1). On the contrary, the low pore volume of SiCoPF sample appears to be related with the high size of the silane precursor, which not only react with surface hydroxyl groups but also block some pores.

Palladium was then incorporated to these modified substrates in order to obtain the catalysts. Palladium loading is similar in both non-functionalized silica Pd-SiCo and Pd-SiCoCl silylated substrates (Table 2), but lower for Pd-SiCoPF homologue. The lower Pd-loading observed for this later sample is associated, as commented above, with the changes in pore volume during silylation of SiCoPF. Because a fraction of the pores of this substrate became blocked, less sulfonic groups capable to be exchanged with palladium species during the metal incorporation are available, and hence a lower amount of palladium is incorporated to this catalyst.

The chemical state of the palladium atoms present in the surface layer of these catalysts was analyzed by X-ray photoelectron spectroscopy (XPS). The photoelectron spectra of the Pd $3 d$ core-levels showed the characteristic spin-orbit splitting of Pd 3d levels, with the most intense $\operatorname{Pd} 3 \mathrm{~d}_{5 / 2}$ component being located at lower binding energies and the least intense $3 \mathrm{~d}_{3 / 2}$ one located at higher binding energies. Although chemical information can be extracted from each of these components, attention is only paid to the most intense one $\left(\operatorname{Pd} 3 d_{5 / 2}\right)$. This $\operatorname{Pd} 3 \mathrm{~d}_{5 / 2}$ peak is asymmetric and placed at relatively high BEs values indicating not only that palladium is oxidized but also that more than one palladium species is present. By applying peak fitting procedures two components are derived (Table 2). The highest BE component $(338.2 \mathrm{eV})$ corresponds to $\mathrm{Pd}^{\mathrm{II}}$ ions interacting with the $-\mathrm{SO}_{3} \mathrm{H}$ groups of the silica, and a second at lower BE (336.5 eV) comes from PdO clusters deposited on the silica surface [11]. The intensity ratio of these components depends on the samples. In agreement with this assignment, the highest intensity of $338.2 \mathrm{eV}$ component is observed for Pd-SiCo sample (Table 2) in which practically all palladium is interacting with $-\mathrm{SO}_{3} \mathrm{H}$ groups. On the contrary, catalysts prepared using the modified supports (Pd-SiCoCl and Pd-SiCoPF) show 
less amount of $\mathrm{Pd}^{\mathrm{II}}$ ions interacting with $-\mathrm{SO}_{3} \mathrm{H}$ than in Pd-SiCo sample (Table 2). In addition to this general trend, some differences are observed among $\mathrm{Pd}-\mathrm{SiCoCl}$ and $\mathrm{Pd}-\mathrm{SiCoPF}$ samples. For Pd-SiCoCl sample the intensity ratio between $338.2 \mathrm{eV}$ and $336.5 \mathrm{eV}$ components is slightly lower than in $\mathrm{Pd}-\mathrm{SiCo}$, and clearly the most intense component corresponds belongs to $\mathrm{Pd}^{\mathrm{II}}$ ions interacting with $-\mathrm{SO}_{3} \mathrm{H}$ groups species. Conversely, the $\mathrm{Pd}$ SiCoPF catalyst shows the main component at $336.5 \mathrm{eV}$ (85\%) which is due, as stated above, $\mathrm{PdO}$ clusters. As in this later sample some pores became blocked during the silylation treatment, less sulfonic groups can exchange palladium species and $\mathrm{Pd}$ moieties are incorporated as PdO clusters. It is important emphasize here that the catalysts with a higher amount of $\mathrm{Pd}^{\mathrm{II}}$ ions interacting with $-\mathrm{SO}_{3} \mathrm{H}$ groups show a high selectivity to $\mathrm{H}_{2} \mathrm{O}_{2}$ in direct synthesis of hydrogen peroxide $[11,12]$.

Catalysts were tested in the direct synthesis of hydrogen peroxide. The hydrogen conversion was very high for all samples ( $>95 \%$ ). The $\mathrm{H}_{2} \mathrm{O}_{2}$ concentration profiles show an increase in the hydrogen peroxide concentration with time (Figure 3). The $\mathrm{H}_{2} \mathrm{O}_{2}$ concentration is higher for Pd-SiCo catalyst at any reaction time than silylated samples. The behavior of Pd-SiCoCl catalyst is strange: no hydrogen peroxide is observed at short reaction times, however after 15 min the $\mathrm{H}_{2} \mathrm{O}_{2}$ concentration grows linearly with a constant slope. The slope of $\mathrm{H}_{2} \mathrm{O}_{2}$ concentration profile for $\mathrm{Pd}-\mathrm{SiCo}$ is clearly higher than for $\mathrm{Pd}-\mathrm{SiCoPF}$ and $\mathrm{Pd}-\mathrm{SiCoCl}$ catalysts. However, after 15 min reaction time $\mathrm{H}_{2} \mathrm{O}_{2}$ concentration slopes for Pd-SiCo and Pd$\mathrm{SiCoCl}$ catalysts are similar.

Selectivity profiles to hydrogen peroxide show a general trend: selectivity deceases with hydrogen peroxide concentration. The catalyst prepared with a non-silylated support shows a high selectivity at low hydrogen peroxide concentration although it decreases slowly to $65 \%$, but it is still higher for all $\mathrm{H}_{2} \mathrm{O}_{2}$ concentrations than with silylated catalysts (Pd-SiCoCl and Pd-SiCoPF). The selectivity trend of $\mathrm{Pd}-\mathrm{SiCoCl}$ catalyst differs markedly from the others because for low $\mathrm{H}_{2} \mathrm{O}_{2}$ concentrations the selectivity is very low, then increasing with the hydrogen peroxide concentration up to $37 \%$. The behavior of this catalyst is related with the high formation rate of water at the beginning of the reaction whereas it becomes reduced to some extent when $\mathrm{H}_{2} \mathrm{O}_{2}$ appears in the reaction medium. Selectivity was recalculated eliminating the initial water formation and the value obtained is clearly higher and is located between Pd-SiCo and Pd-SiCoPF catalysts. 
Activity data are in good agreement with the nature of the Pd species, as revealed by XPS. The catalyst with the lowest proportion of $\mathrm{PdO}$, and hence with the highest amount of $\mathrm{Pd}^{\mathrm{II}}$ ions interacting with $-\mathrm{SO}_{3} \mathrm{H}$ groups ( $\mathrm{Pd}-\mathrm{SiCo}$ ), yields the highest selectivity and $\mathrm{H}_{2} \mathrm{O}_{2}$ concentration. Pd-SiCoCl catalyst has an intermediate behavior whereas Pd-SiCoPF catalyst produces the lowest selectivity and $\mathrm{H}_{2} \mathrm{O}_{2}$ concentration. The absence of hydrogen peroxide observed at short reaction time using $\mathrm{Pd}-\mathrm{SiCoCl}$ catalysts can be related with the homogeneous silylation obtained for this sample. A hydrophobic surface has been developed in this catalyst which makes it non-wetable by a highly polar solvent (methanol), and hence strong mass transfer resistance for reactants and products is developed at the catalyst surface.

In general, reaction data are difficult to compare with those published in open literature because reaction conditions are different. In the present work, we have used a reaction temperature of $313 \mathrm{~K}$, which is much more attractive from an industrial perspective and differs substantially from that used in most published works (273-278 K). The higher temperature used in this work has a two-fold effect: (i), a high reaction temperature determines a high reaction rate, and therefore much higher hydrogen peroxide concentrations are obtained at shorter reaction times (7-8 wt \% in this work), which contrasts with the much lower concentrations usually reported in the literature; and (ii), the reaction temperature of $313 \mathrm{~K}$ selected in this work is closer to industrial application because the reaction $\mathrm{H}_{2}+\mathrm{O}_{2} \rightarrow$ $\mathrm{H}_{2} \mathrm{O}_{2}$ is highly exothermic and hence the heat released must be removed, this being easier at $313 \mathrm{~K}$ than at $278 \mathrm{~K}$.

\section{Conclusions}

The influence of chemical modification of Pd-based catalysts on the performance for hydrogen peroxide direct synthesis was studied in this work. Silylation of the support led to a decrease in the BET specific area, but other changes in textural properties were found to depend on the silylating agent used. Hydrophobicity of treated samples was confirmed by TGA, showing a decrease in the adsorbed water on silylated samples. These silylated substrates were then used to prepare catalysts by ionic exchange with a palladium salt. In all cases, palladium was incorporated as exchanged ( $\mathrm{Pd}^{\mathrm{II}}$ species) and $\mathrm{PdO}$ clusters, the ratio of these species depends on the support employed. In the photoelectron spectra of the samples, the highest proportion of the $\mathrm{Pd} 3 \mathrm{~d}_{5 / 2}$ component at $338.2 \mathrm{eV}$ corresponded to Pd-SiCo sample, in which almost all palladium incorporated interacts with $-\mathrm{SO}_{3} \mathrm{H}$ groups. Catalysts prepared 
with modified supports (Pd-SiCoCl and Pd-SiCoPF) showed less amount of $\mathrm{Pd}^{\mathrm{II}}$ ions interacting with $-\mathrm{SO}_{3} \mathrm{H}$ than $\mathrm{Pd}-\mathrm{SiCo}$. Catalyst system of supported palladium nanoparticles deposited on $\mathrm{HSO}_{3}$-functionalized commercial silica produced hydrogen peroxide with a high yield by feeding a non-flammable $\mathrm{H}_{2} / \mathrm{O}_{2}$ mixture into a non-acidic catalyst-methanol suspension at $313 \mathrm{~K}$. However, catalysts prepared with silylated supports show a low yield to hydrogen peroxide, due to low $\mathrm{H}_{2} \mathrm{O}_{2}$ selectivity. The drop in the selectivity to hydrogen peroxide is related with the different proportion of palladium species present in silylated catalysts as compared to non silyled sample.

\section{Acknowledgements}

The authors acknowledge financial support from Repsol-YPF (Spain) and the Spanish Ministry of Science and Education in the projects PSE-310200-2006-2 and FIT-320100-200688. GBB gratefully acknowledge fellowships granted by Repsol-YPF.

\section{References}

1. J.M. Campos-Martin, G. Blanco-Brieva and J.L.G. Fierro, Angew. Chem. Int. Ed. 45 (2006), 6962.

2. G. Goor, W. Kunkel, O. Weiberg, Ullmann's Encyclopedia of Industrial Chemistry, in: B. Elvers, S. Hawkins, M. Ravenscroft, G. Schulz, VCH, Weinheim (Eds.), New York, Vol. A13 1989 pp. 443-466.

3. H. Henkel, W. Weber, US Patent 1108752 (1914).

4. $\quad$ L.W. Gosser, US Patent 4681751 (1988).

5. R. Burch and P.R. Ellis, Appl. Catal. B: Environ. 42 (2003) 203.

6. C. Samanta and V. R. Choudhary, Applied Catalysis A: General 330 (1-2) (2007), 23

7. S. Chinta and J.H. Lunsford, J. Catal. 225 (2004) 249.

8. Q. Liu and J.H. Lunsford, J. Catal. 239 (2006) 237.

9. D. P. Dissanayake and J. H. Lunsford, J. Catal. 206 (2002) 173

10. D.P. Dissanyake and J.H. Lunsford, J. Catal. 214 (2003) 113 
11. G. Blanco-Brieva, E. Cano-Serrano, J.M. Campos-Martin and J.L.G. Fierro, Chem. Commun. (2004) 1184

12. C. Burato, P. Centomo, M. Rizzoli, A. Biffis, S. Campestrini and B. Corain, Adv. Synth. Catal., 348 (2006) 255

13. G. Blanco-Brieva, M. C. Capel-Sanchez, M. P. de Frutos, J. M. Campos-Martin and, J. L. G. Fierro, Ind. Eng. Chem. Res., 47 (2008) 8013.

14. B. Corain and M. Kralik, J. Mol. Catal. A, 173 (2001) 99.

15. E. Cano-Serrano, J. M. Campos-Martin and J. L. G. Fierro, Chem.Commun., 9 (2) (2003) 246.

16. E. Cano-Serrano; G, Blanco-Brieva; J. M. Campos-Martin and; J. L. G. Fierro, Langmuir, 19 (2003) 7621.

17. G. Blanco-Brieva, E. Cano-Serrano, J.M. Campos-Martin and J.L.G. Fierro EP 2000205 (2009)

18. J. K. Edwards, B. Solsona, E. Ntainjua, A. F. Carley, A. A. Herzing, C. J. Kiely and G. J. Hutchings, Science, 323 (5917) (2009) 1037.

19. N. R. E. N. Impens, P. van der Voort and E. F. Vansant, Microp. Mesop. Mater, 28 (1999) 217.

20. M.C. Capel-Sanchez, L. Barrio, J.M. Campos-Martin, J.L.G. Fierro, J. Coll. Interface Sci. 277 (2004) 146

21. W. Weici, W. Xiaolong, W. Daoai, C. Miao, Z. Feng, L. Weimin and X. Qunji, Chem. Commun, (2009) 1043-1045.

22. C.D. Wagner, W.M. Riggs, L.E. Davis, J.F. Moulder and G.E. Muilenberg, Handbook of X-ray Photoelectron Spectroscopy, Perkin Elmer Corp. (1979).

23. K. S. W. Sing, D. H. Everett, R. A.W. Haul, L. Moscou, R. A. Pierotti, J. Rouquerol, T. Siemieniewska, Pure Appl. Chem. 75 (1985) 603. 
Table 1. Supports prepared and textural properties.

\begin{tabular}{|c|c|c|c|}
\hline Support & Silylating agent & $\begin{array}{c}\text { BET area } \\
\left(\mathbf{m}^{\mathbf{2}} \mathbf{g}^{-\mathbf{1}}\right)\end{array}$ & $\begin{array}{c}\text { Pore volume } \\
\left(\mathbf{m} \mathbf{L g}^{-1}\right)\end{array}$ \\
\hline $\mathrm{SiCo}$ & --- & 251 & 0.38 \\
\hline $\mathrm{SiCoCl}$ & $\left(\mathrm{CH}_{3}\right)_{3} \mathrm{SiCl}$ & 201 & 0.42 \\
\hline $\mathrm{SiCoPF}$ & $\mathrm{CF}_{3}\left(\mathrm{CF}_{2}\right)_{5}\left(\mathrm{CH}_{2}\right)_{2} \mathrm{Si}\left(\mathrm{CH}_{3}\right)_{2} \mathrm{Cl}$ & 201 & 0.37 \\
\hline
\end{tabular}

Table 2. Catalyst prepared, palladium concentration determined by chemical analysis and $\mathrm{BE}$ of $\mathrm{Pd} 3 \mathrm{~d}$ region of catalysts.

\begin{tabular}{|c|c|c|c|}
\hline Catalyst & Support & Pd (wt. \%) & $\operatorname{Pd} 3 d_{5 / 2}$ BE $(\mathrm{eV})$ \\
\hline $\mathrm{Pd}-\mathrm{SiCo}$ & SiCo & 1.9 & $\begin{array}{l}336.5(10) \\
338.2(90)\end{array}$ \\
\hline $\mathrm{Pd}-\mathrm{SiCoCl}$ & $\mathrm{SiCoCl}$ & 1.9 & $\begin{array}{l}336.5(20) \\
338.2(80)\end{array}$ \\
\hline Pd-SiCoPF & SiCoPF & 1.4 & $\begin{array}{l}336.5(85) \\
338.2(15)\end{array}$ \\
\hline
\end{tabular}

${ }^{*}$ Relative ratio of each component in parenthesis. 


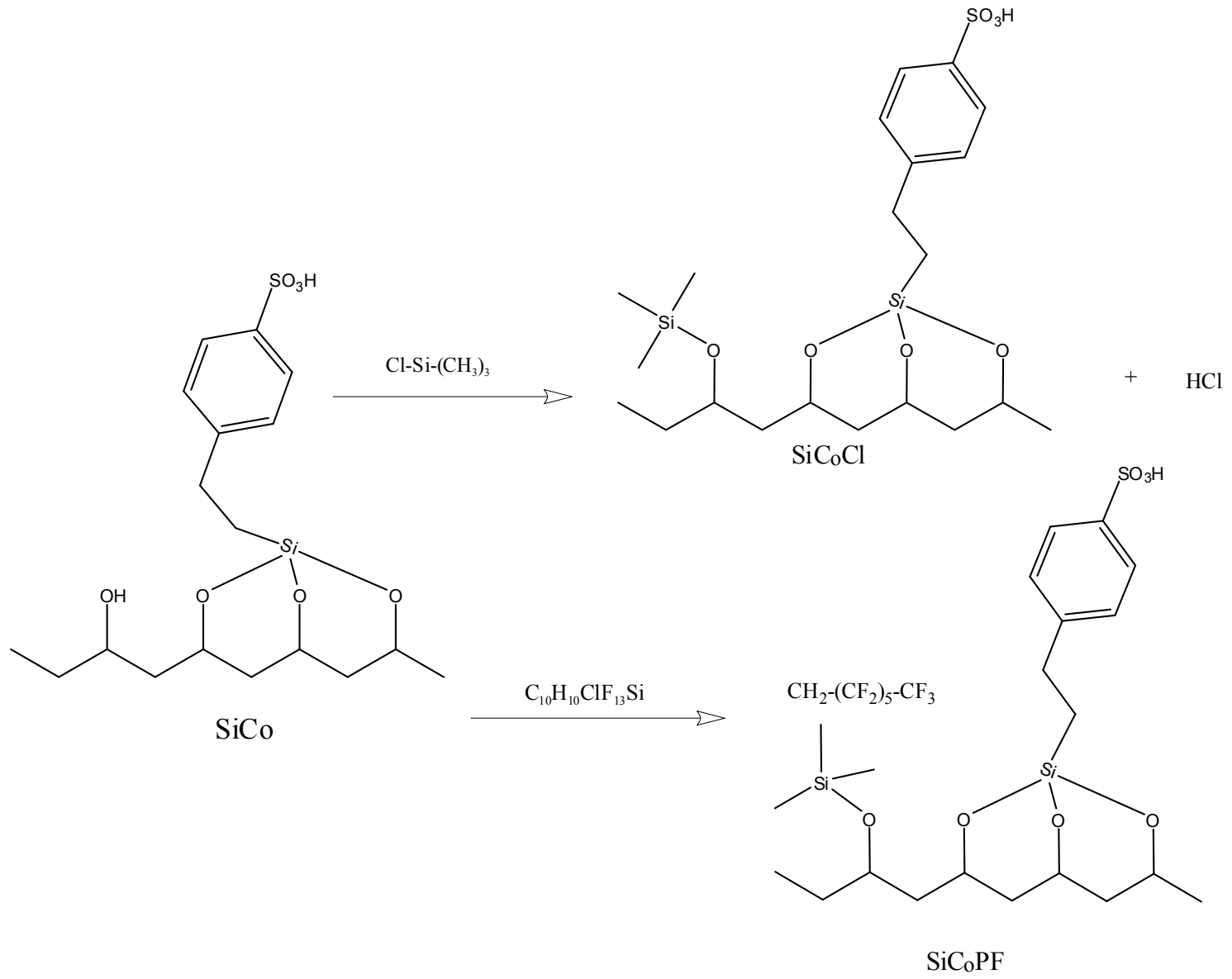

Scheme 1. Schematic modification of the silica functionalized support. 

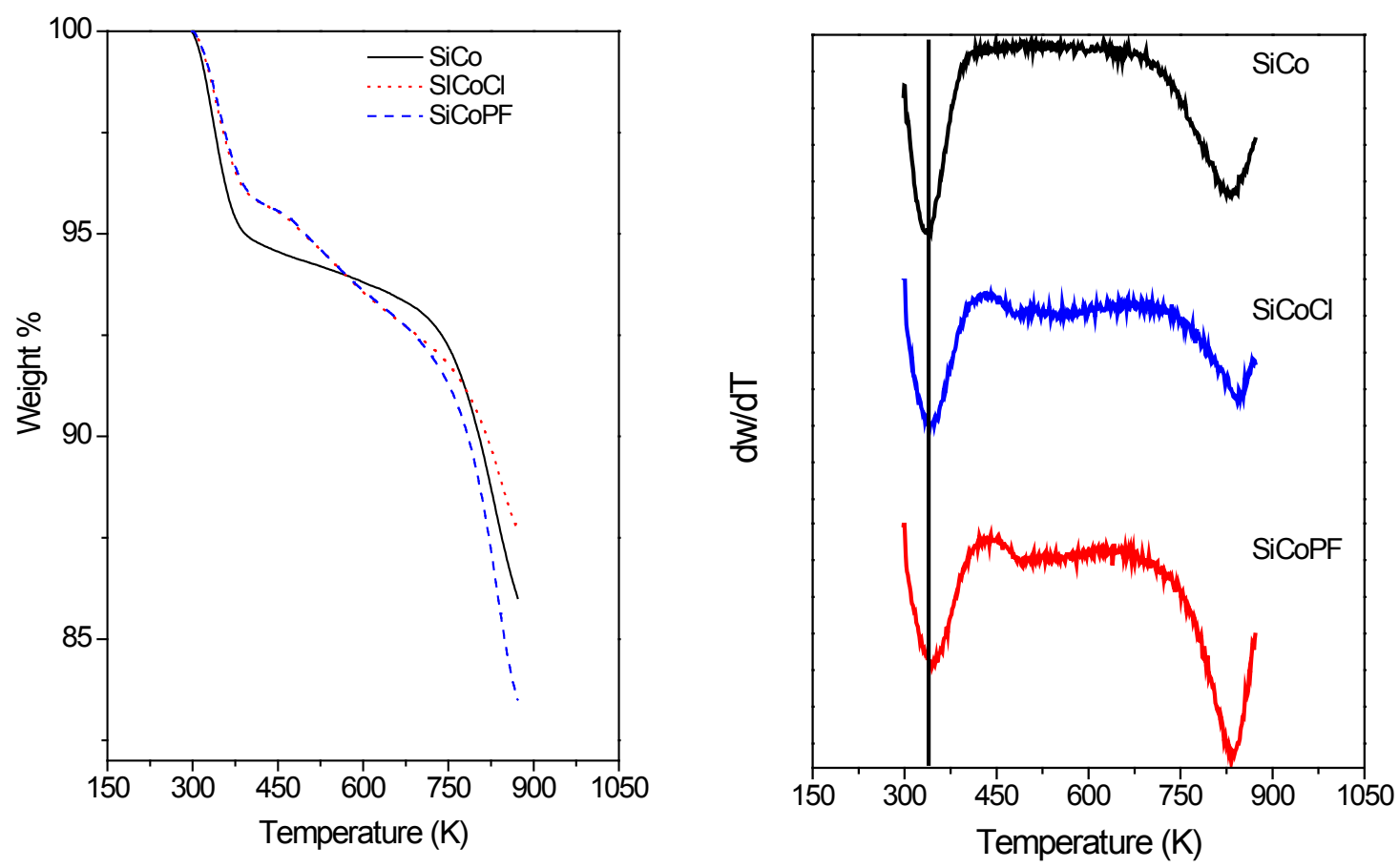

Figure 1 TG and DTG profiles of the studied supports. 

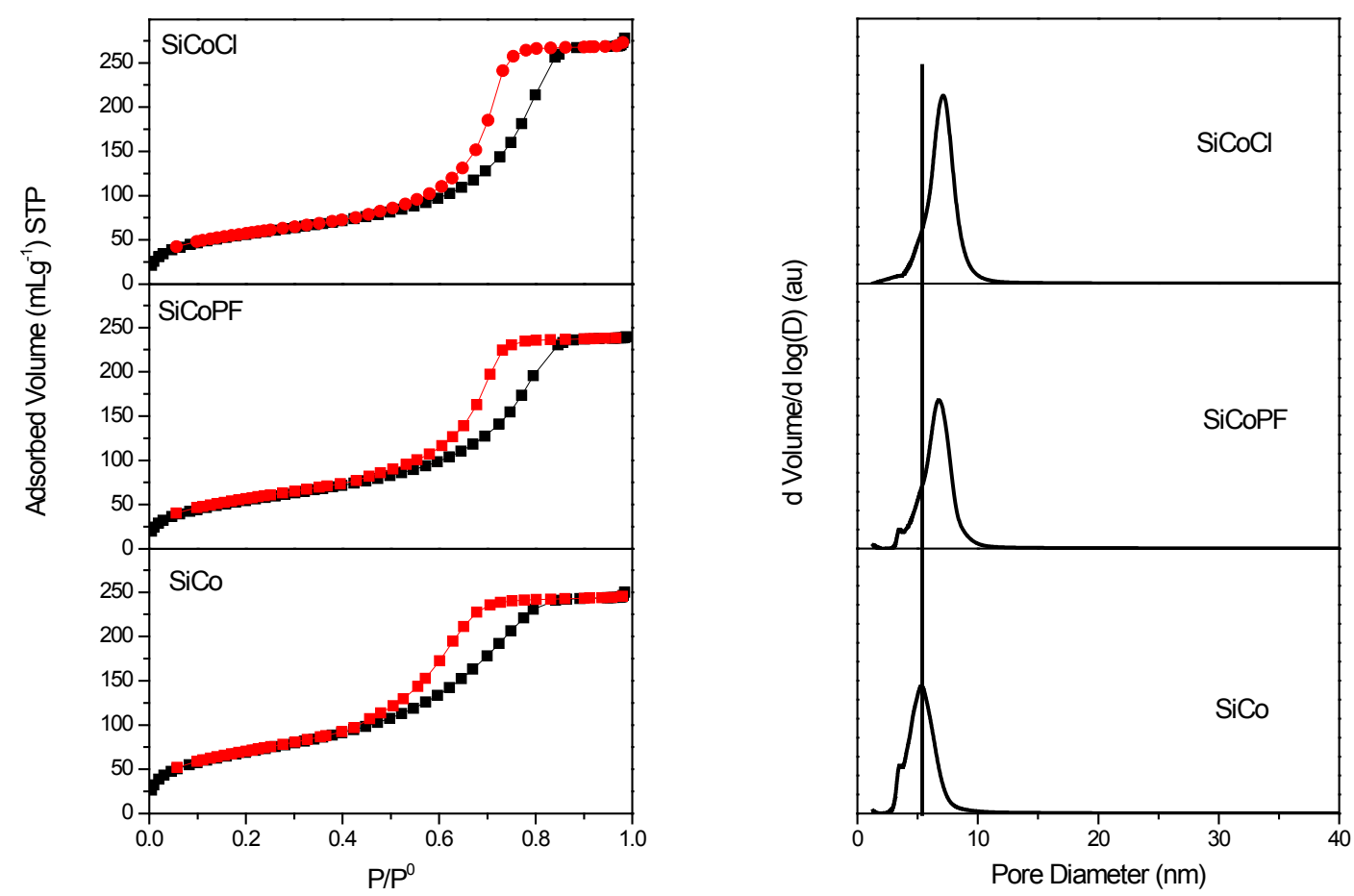

Figure 2 Nitrogen adsorption isotherms at $77 \mathrm{~K}$ and pore size distribution of samples derived by applying the BJH method to the desorption branch. 

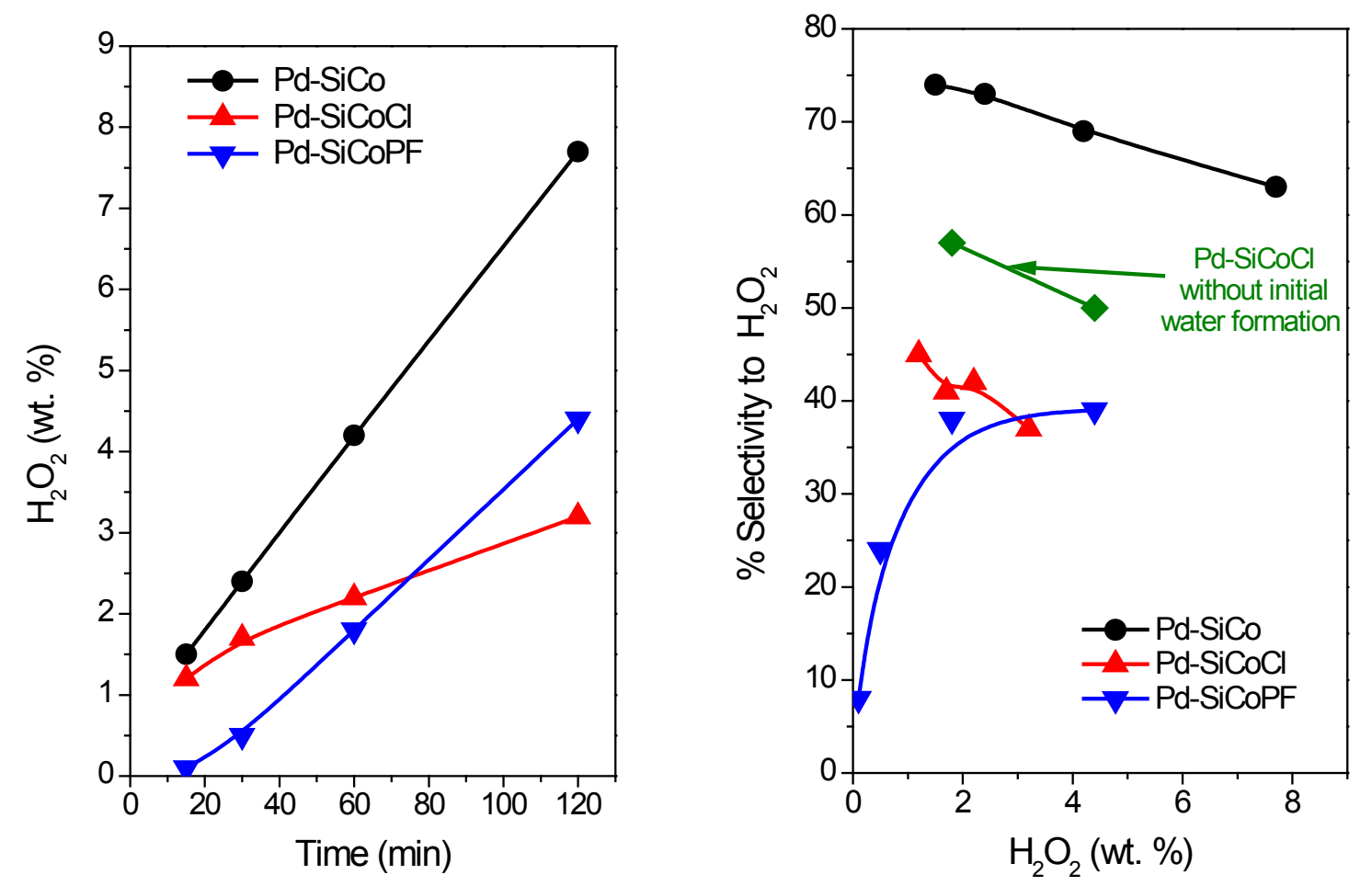

Figure $3 \quad \mathrm{H}_{2} \mathrm{O}_{2}$ concentration and selectivity profile versus time during direct synthesis of hydrogen peroxide at $313 \mathrm{~K}$. 